TITLE:

\title{
Partitioning a Polygonal Region into Trapezoids
}

$\operatorname{AUTHOR}(\mathrm{S})$ :

ASANO, Takao; ASANO, Tetsuo; IMAI, Hiroshi

\section{CITATION:}

ASANO, Takao ...[et al]. Partitioning a Polygonal Region into Trapezoids. 数理解析研究所 講究録 1984, 522: 26-52

\section{ISSUE DATE:}

1984-05

URL:

http://hdl.handle.net/2433/98478

RIGHT: 
***Department of Mathematical Engineering and Instrumentation Physics, Faculty of Engineering, University of Tokyo, Bunkyo-ku, Tokyo, Japan 113

***Faculty of Engineering, Osaka Electro-Communication University, Hatsu-cho, Neyagawa, Osaka, Japan 572

February, 1984

\begin{abstract}
.
The problem of partitioning a polygonal region into a minimum number of trapezoids with two horizontal sides is discussed. A triangle with a horizontal side is considered to be a trapezoid with two horizontal sides one of which is degenerate. First, a method of achieving a minimum partition is presented. The number $M *$ of the trapezoids in the minimum partition of a polygonal region $P$ is shown to be $M *=n+w-h-d-1$, where $n$, $w$, and $h$ are the number of vertices, windows, and horizontal edges of $P$, respectively, and $d$ is the cardinality of a maximum independent set of the straight-lines-in-the-plane graph associated with P. Next, this problem is shown to be polynomially equivalent to the problem of finding a maximum independent set of a straight-lines-in-the-plane graph, and consequently, it is shown to be NP-complete. However, for a polygonal region without windows, an $0\left(\mathrm{n}^{2}\right)$ time algorithm for partitioning it into a minimum number of trapezoids is presented. Finally, an $O(n \log n)$ time approximation algorithm with absolute performance ratio 3 is presented.
\end{abstract}

*This paper is an expanded version of a paper [1] presented at the 24th Annual IEEE Symposium on the Foundations of Computer Science, Tucson, 1983. 


\section{Introduction}

The problem of partitioning a geometric figure into a minimum number of more fundamental ones arises in applications such as manipulation of VLSI artwork data, image processing, and architectural data base. Triangles, convex polygons, and rectangles have been chosen to be the fundamental figures. For a simple polygon (i.e., a polygonal region, without holes), there have been several polynomial-time algorithms for partitioning it into a minimum number of triangles, convex polygons, etc. $[3,4,8,16]$. However, for a polygonal region with holes, in most cases, this problem has been shown to be NP-complete $[13,18]$. On the other hand, Lipski et al. [19] and Ohtsuki [20] gave polynomial-time algorithms for partitioning a rectilinear polygonal region into a minimum number of rectangles.

In this paper, we consider the problem of partitioning a polygonal region into a minimum number of trapezoids with two horizontal sides. A triangle with a horizontal side is considered to be a trapezoid with two horizontal sides one of which is degenerate. This problem is closely related to VLSI artwork data processing systems of Electron-Beam Lithography for VLSI microfabrication which have been becoming in use. In such systems, the layout is stored as a set of polygonal regions per layer. These regions are bounded by straight lines which may have any slope. They may contain windows, or "polygon holes". Each polygonal region should be partitioned into fundamental figures since the aperture of a pattern generator is restricted. Trapezoids are used as fundamental figures in AMDES [21], one of EB Lithography systems. Evident1y, the processing time is proportional to the number of trapezoids. Thus a minimum partition of a polygonal region is required.

First, we describe the problem in graph-theoretic terms, and present a method of achieving a minimum partition of a polygonal region $P$ into trapezoids. The number $M *$ of the trapezoids in the minimum partition is shown to be $M^{*}=n+w-h-d-1$, where $n, w$, and $h$ are the number of vertices, windows, and horizontal edges of $\mathrm{P}$, respectively, and $\mathrm{d}$ is the cardinality of a maximum independent set of the straight-lines-in-the-plane graph associated with $P$. Next, we show that this problem is polynomially equivalent to the problem of finding a maximum independent set of a straight-1ines-in-the-plane graph. Thus, it is shown to be NP-complete.

Although it is NP-complete to partition a polygonal region into a minimum number of trapezoids, there may be a polynomial-time algorithm for 
a polygonal region with a fixed number of windows. In fact, we present an $\mathrm{O}\left(\mathrm{n}^{2}\right)$ time algorithm for partitioning a polygonal region without windows into a minimum number of trapezoids. Also, we give an $0\left(n^{2+w}\right)$ time algorithm for a polygonal region with w windows.

Finally, we present an $O(n \log n)$ time approximation algorithm which uses only horizontal chords to partition a polygonal region into trapezoids. We show that the absolute performance ratio of the algorithm is 3 for polygonal regions ( 2 for polygonal regions without windows). We can design another approximation algorithm with the absolute performance ratio $(1+2 / c)$ for polygonal regions $((1+1 / c)$ for polygonal regions without windows) if we have a $(1-1 / c)$ approximation algorithm for the maximum independent set problem on straight-lines-in-the-plane graphs, where $c$ is some constant. From the viewpoint of practical applications, a minimum partition is not so important. A near optimal partition is quite satisfactory in practice.

\section{Preliminaries}

In this section we present some definitions and results necessary for the discussions in the following sections.

Consider a set of pairwise non-intersecting simple polygons $\mathrm{W}_{0}, \mathrm{~W}_{1}$, $\ldots, W_{W}$. If one polygon, say $W_{0}$, contains all other polygons $W_{1}, \ldots, W_{W}$ and no other polygon contains any polygons, then we have the connected region specified by the interior region of $\mathrm{W}_{0}$ excluding the interior regions of $W_{1}, \ldots, W_{w}$. Such a connected region is called a polygonal region of one connected region and is illustrated in Fig. $1 . \mathrm{W}_{0}$ is called the external polygon and $W_{1}, \ldots, W_{W}$ are called internal polygons. Internal polygons are often called windows or holes. Thus, a polygonal region is specified by the set of its polygon edges (edges of polygons of the polygonal region). Each polygon edge is oriented in a way that the interior lies to the right of the edge. In other words, the external polygon is oriented in clockwise order, whereas the internal polygons (windows) are oriented in counter-clockwise order. Any three consecutive vertices must not be collinear. If any, we eliminate consecutive collinear vertices. We consider only a polygonal region of exactly one connected region. (A polygonal region with several connected regions is considered to be a set of polygonal regions with one connected region.)

Given a polygonal region, we must add chords to partition it into trapezoids. In this paper, only trapezoids with two horizontal sides are 


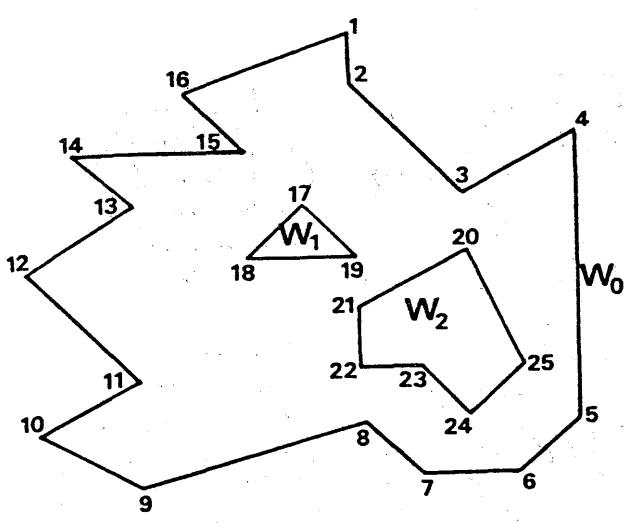

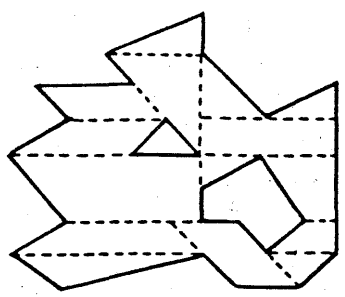

(a)

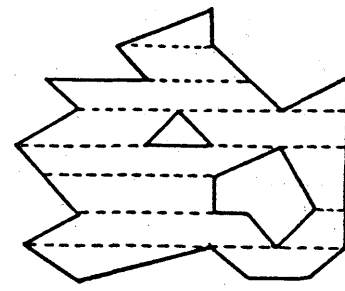

(b)

Fig. 2. Partition of $P$ into trapezoids.

(a) Non-optimal partition. (b) Optimal partition.

Fig. 1. A polygonal region $P$ with two windows $W_{1}$ and $W_{2}$ and the external polygon $W_{0}$.

considered to be trapezoids. A triangle with a horizontal side is considered to be a trapezoid one of whose horizontal sides is degenerate. Partitions into trapezoids are shown in Fig. 2. Fig. 2(a) shows a non-optimal partition while Fig. 2(b) an optimal one, where an optimal partition means a partition into a minimum number of trapezoids. In the following, we shall investigate effects of chords for partitioning a polygonal region into trapezoids.

Definition 1. A chord of a polygonal region is a straight line segment lying inside it. Each of its endpoints must be a vertex or lie on a polygon edge. It may touch vertices on the way, but it must not intersect with any edges. A chord is primitive if it touches no vertices except at the endpoints.

Definition 2. Let $s$ be a primitive chord of a polygonal region $P$. Then the resultant region(s) after adding it to $\mathrm{P}$ is denoted by $\mathrm{P}+\mathrm{s}$. If its endpoints both lie on the external polygon of $\mathrm{P}$ or both on the same window, then $\mathrm{P}$ is decomposed into two regions (see Fig. 3(a) and (b)). Thus, $\mathrm{P}+\mathrm{s}$ consists of two distinct polygonal regions. If one endpoint lies on the external polygon and the other on a window, then the window is eliminated and connected to the external polygon (see Fig. 3(c)). If two endpoints lie on two distinct windows, then these two windows are merged into one (see Fig. 3(d)).

Definition 3. Let $s_{1}$ be a primitive chord of a polygonal region $P$ and $\mathrm{s}_{2}$ be a primitive chord of $\mathrm{P}+\mathrm{s}_{1}$ (i.e., $\mathrm{s}_{2}$ is a primitive chord of some polygonal region in $\mathrm{P}+\mathrm{s}_{1}$ ), then $\mathrm{P}+\mathrm{s}_{1}+\mathrm{s}_{2}$ is defined to be the polygonal region(s) obtained from $\mathrm{P}$ by adding $\mathrm{s}_{1}$ and then $\mathrm{s}_{2}$ to $\mathrm{P} . \mathrm{P}_{1}+\mathrm{s}_{1}+\mathrm{s}_{2}+\ldots+\mathrm{s}_{\mathrm{m}}$, 


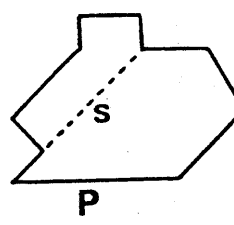

(a)
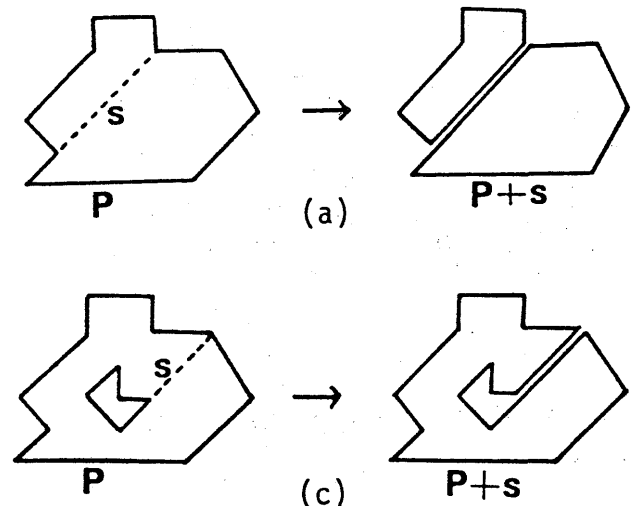

(c)

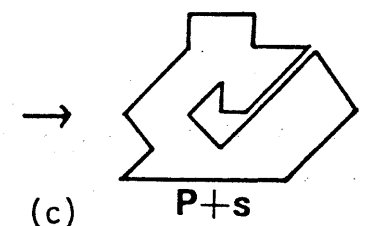

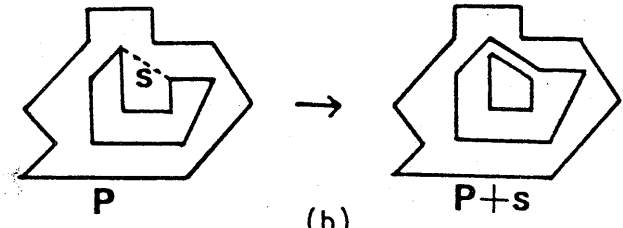

(b)

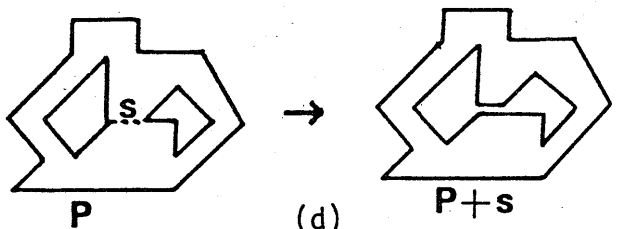

Fig. 3. Resultant polygonal region(s) $P+s$ after adding a primitive chord $s$ to a polygonal region $P$. (a) Both endpoints lie on the external polygon. (b) Both endpoints lie on the same window.

(c) One endpoint lies on the external polygon and the other on a window.

(d) Two endpoints lie on distinct windows.

$m \geq 3$, is defined in a similar way. For a nonprimitive chord $\mathrm{s}$, such as shown in Fig. 4, which consists of primitive collinear chords $s_{1}, s_{2}, \ldots$, $\mathrm{s}_{\mathrm{m}}, \mathrm{P}+\mathrm{s}$ is defined to be $\mathrm{P}+\mathrm{s}_{1}+\mathrm{s}_{2}+\ldots+\mathrm{s}_{\mathrm{m}}$.

Since any connected planar graph with $\mathrm{p}$ nodes, $\mathrm{q}$ arcs and $\mathrm{f}$ faces satisfies $\mathrm{p}-\mathrm{q}+\mathrm{f}=2$ (Euler's formula [9]), we have the following lemma.

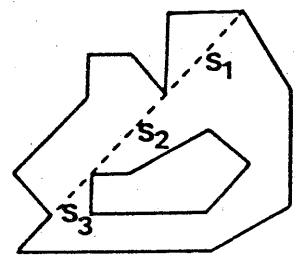

Fig. 4. A non-primitive chord $s$ which consists of three primitive chords $s_{1}, s_{2}$ and $s_{3}$.

Lemma 1. Let $\mathrm{D}$ be the number of pairwise non-intersecting primitive chords drawn to partition a polygonal region $P$ into trapezoids. Then, the number $M(P)$ of trapezoids is given by

$$
M(P)=D-w(P)+1,
$$

where $w(P)$ is the number of windows contained in $P$.

An index of a polygonal region in the following definition plays the most important role in this paper.

Definition 4. Let $P$ be a polygonal region. Then, an index $t(P)$ of $P$ is defined by

$$
t(P)=n(P)+w(P)-h(P)-1,
$$

where $n(P), w(P)$, and $h(P)$ represent the number of vertices, windows, and horizontal edges of $P$, respectively. Similarly, an index $t(\Phi)$ of a set of polygonal regions $\Phi=\left\{\mathrm{P}_{1}, \mathrm{P}_{2}, \ldots, \mathrm{P}_{\mathrm{k}}\right\}$ is defined by the sum of all indices of the polygonal regions in $\Phi$, i.e.,

$$
t(\Phi)=\sum_{i=1}^{k} t(P) .
$$


Evidently, the index of any trapezoid with two horizontal sides is one. Thus we have the following lemma.

Lemma 2. If $\Phi$ is a set of trapezoids with two horizontal sides, then the index $t(\Phi)$ represents the number of those trapezoids.

In other words, if a sequence of chords $\left(s_{1}, s_{2}, \ldots, s_{m}\right)$ partitions a polygonal region $P$ into trapezoids, then the index $t\left(P+s_{1}+s_{2}+\ldots+s_{m}\right)$ represents the number of those trapezoids.

Definition 5. Let $s$ be a chord of a polygonal region $P$. An effect of $S$ in $P$ is defined by

$\operatorname{effect}(s ; P)=t(P)-t(P+s)$. Generally, an effect of a chord $\mathrm{s}_{\mathrm{k}}$ in $\mathrm{P}+\mathrm{s}_{1}+\ldots+\mathrm{s}_{\mathrm{k}-1}$ is defined by $\operatorname{effect}\left(\mathrm{s}_{\mathrm{k}} ; \mathrm{P}+\mathrm{s}_{1}+\ldots+\mathrm{s}_{\mathrm{k}-1}\right)=\mathrm{t}\left(\mathrm{P}+\mathrm{s}_{1}+\ldots+\mathrm{s}_{\mathrm{k}-1}\right)-\mathrm{t}\left(\mathrm{P}+\mathrm{s}_{1}+\ldots+\mathrm{s}_{\mathrm{k}}\right)$.

Let $\left(s_{1}, s_{2}, \ldots, s_{m}\right)$ be a sequence of chords which partitions a polygonal region $P$ into trapezoids. Then, we have

$t\left(P+s_{1}+\ldots+s_{m}\right)=t(P)-\sum_{k=1}^{m} \operatorname{effect}\left(s_{k} ; P+s_{1}+\ldots+s_{k-1}\right)$. Therefore, a sequence of chords to maximize the sum of their effects partitions $P$ into a minimum number of trapezoids. The following lemmas are concerned with the effect of a chord s.

Lemma 3. Let $s$ be a primitive chord of a polygonal region $P$. Then, $-3 \leq \operatorname{effect}(\mathrm{s} ; \mathrm{P}) \leq 1$. Specifically, the following hold.

(a) Effect(s;P)=1 if and only if the following (i) or (ii) holds.

(i) $\mathrm{s}$ is a horizontal chord between two vertices of $\mathrm{P}$.

(ii) Two edges collinear with $\mathrm{s}$ are adjacent to $\mathrm{s}$.

(b) Effect(s;P)=0 if and only if the following (iii), (iv) or (v) holds.

(iii) $s$ is a horizontal chord and exactly one of its ednpoints is a vertex of $P$.

(iv) $\mathrm{s}$ is a non-horizontal chord between two vertices of $\mathrm{P}$ and exactly one of edges adjacent to $s$ is collinear with $s$.

(v) $\mathrm{s}$ is a non-horizontal chord between a vertex of an edge collinear with $\mathrm{S}$ in $\mathrm{P}$ and a point on a horizontal edge of $\mathrm{P}$ which is not a vertex of $P$.

(c) Effect $(s ; P)$ is negative if and only if $s$ does not satisfy any condition stated above.

Proof. Let $\alpha$ be the number of those endpoints of $s$ which are not vertices of $P$, and $\beta$ be the number of those polygon edges adjacent to $s$ which are collinear with $s$. Let $\gamma$ be the number of those endpoints of $s$ 


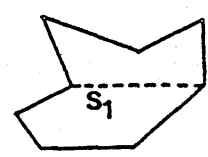

(i)

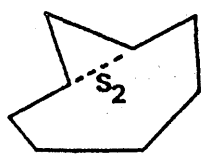

(ii)

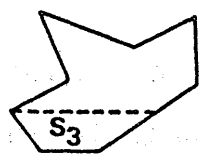

(iii)

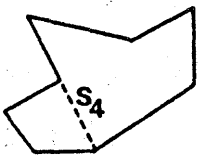

(iv)

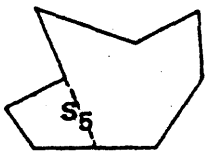

(v)

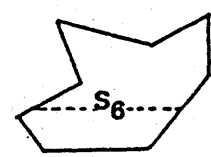

(vi)

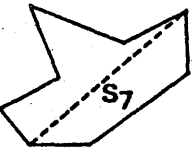

(vii)

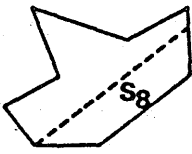

(viii)

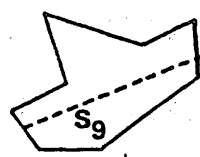

(ix)

Fig. 5. Effects of chords. (i) effect $\left(s_{1} ; P\right)=1$. (ii) $\operatorname{effect}\left(s_{2} ; P\right)=1$.

(iii) $\operatorname{effect}\left(s_{3} ; P\right)=0$. (iv) effect $\left(s_{4} ; P\right)=0$. (v)effect $\left(s_{5} ; P\right)=0$.

(vi) effect $\left(s_{6} ; P\right)=-1$. (vii) effect $\left(s_{7} ; P\right)=-1$.

(viii) effect $\left(s_{8} ; P\right)=-2$. (ix) effect $\left(s_{g} ; P\right)=-3$.

which are not vertices of $P$ but are points on horizontal edges of $P$. Then, $0 \leq \gamma \leq \alpha \leq 2$ and $0 \leq \beta \leq 2$, and consequent1y, the proposition in the lemma is equivalent to the following:

$$
\operatorname{effect}(s ; P)= \begin{cases}1-\alpha & \text { if } s \text { is horizontal, } \\ \beta-(\alpha-\gamma)-1 & \text { otherwise. }\end{cases}
$$

Let $\mathrm{n}(\mathrm{P}+\mathrm{s}), \mathrm{w}(\mathrm{P}+\mathrm{s}), \mathrm{h}(\mathrm{P}+\mathrm{s})$, and $|\mathrm{P}+\mathrm{s}|$ denote the number of vertices, windows, horizontal edges, and connected regions, respectively, of the resulting polygonal region(s) $\mathrm{P}+\mathrm{s}$ obtained by adding $\mathrm{s}$ to $\mathrm{P}$. Note that

$$
\begin{aligned}
& t(P)=n(P)+w(P)-h(P)-1, \\
& t(P+s)=n(P+s)+w(P+s)-h(P+s)-|P+s| \text {, and } \\
& \text { effect }(s ; P)=t(P)-t(P+s) .
\end{aligned}
$$

Thus, we can obtain (2.1) by counting $n(P+s), w(P+s), h(P+s)$, and $|P+s|$, according to the location of two endpoints of $s$ (Fig. 3 ), because we have

$$
\begin{aligned}
n(P+s) & =n(P)+2-\beta+\alpha, \\
w(P+s) & = \begin{cases}w(P) & \text { for cases (a) and (b), } \\
w(P)-1 & \text { for cases (c) and (d), }\end{cases} \\
h(P+s) & = \begin{cases}h(P)+2-\beta & \text { if } s \text { is horizontal, } \\
h(P)+\gamma & \text { otherwise, }\end{cases} \\
|P+s| & = \begin{cases}2 & \text { for cases (a) and (b), } \\
1 & \text { for cases (c) and (d). }\end{cases}
\end{aligned}
$$

Fig. 5 illustrates the effects of various chords. In Lemma 3 we require chords to be primitive. Generally, we have the following lemma by a similar argument.

Lemma 4. Let $\mathrm{s}$ be any chord (not necessarily primitive) of a polygonal region $P$. Then, effect $(s ; P)$ is positive if and only if the 
following (i) or (ii) holds.

(i) $s$ is a horizontal chord between two vertices of $P$.

(ii) Two edges collinear with $\mathrm{s}$ are adjacent to s.

Hereafter, we call a chord to be effective if

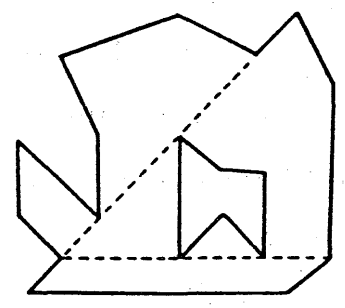
its effect is positive. Fig. 6 shows an example of effective chords which are not primitive. They both. Fig. 6. Effective chords consist of three primitive chords.

which are not primitive.

Lemma 5. Let $s$ be any chord of a polygonal region $P$, and $\operatorname{SEC}(P)$ and $\mathrm{SEC}(\mathrm{P}+\mathrm{s})$ be the sets of all effective chords of $\mathrm{P}$ and $\mathrm{P}+\mathrm{s}$, respectively. Then, the following holds:

$$
|\operatorname{SEC}(\mathrm{P})| \geq|\operatorname{SEC}(\mathrm{P}+\mathrm{s})|+\operatorname{effect}(\mathrm{s} ; \mathrm{P}) \text {. }
$$

Proof. This lemma can be proved in the same way as in the proof of Lemna 3 .

We now present a natural partition method, which is not only simple but also optimal for some polygonal regions.

[Partition method $\mathrm{H}$ ]

For each vertex of a polygonal region, draw as many horizontal chords as possible within the region to the first edge encountered.

Theorem 1. Partition method $\mathrm{H}$ is optimal for a polygonal region $\mathrm{P}$ with no effective chords. The number of trapezoids is given by the index of $P$, that is,

$$
t(P)=n(P)+w(P)-h(P)-1 \text {. }
$$

Proof. Let $\left(\mathrm{s}_{1}, \mathrm{~s}_{2}, \ldots, \mathrm{s}_{\mathrm{m}}\right)$ be any sequence of chords which partitions a polygonal region $P$ into trapezoids. For each $k(k=1,2, \ldots, m)$, let $\operatorname{SEC}\left(\mathrm{P}+\mathrm{s}_{1}+\ldots+\mathrm{s}_{\mathrm{k}}\right)$ be the set of all effective chords of $\mathrm{P}+\mathrm{s}_{1}+\ldots+\mathrm{s}_{\mathrm{k}}$. Then, by Lemma 5 , we have

$$
-\operatorname{effect}\left(\mathrm{s}_{\mathrm{k}} ; \mathrm{P}+\mathrm{s}_{1}+\ldots+\mathrm{s}_{\mathrm{k}-1}\right) \geq\left|\operatorname{SEC}\left(\mathrm{P}+\mathrm{s}_{1}+\ldots+\mathrm{s}_{\mathrm{k}}\right)\right|-\left|\operatorname{SEC}\left(\mathrm{P}+\mathrm{s}_{1}+\ldots+\mathrm{s}_{\mathrm{k}-1}\right)\right| .
$$

Therefore, we have

$$
\begin{aligned}
t\left(P+s_{1}+\ldots+s_{m}\right) & =t(P)-\sum_{k=1}^{m} \operatorname{effect}\left(s_{k} ; P+s_{1}+\ldots+s_{k-1}\right) \\
& \geq t(P)+\left|\operatorname{sEC}\left(P+s_{1}+\ldots+s_{m}\right)\right|-|\operatorname{SEC}(P)| .
\end{aligned}
$$

Since we assumed that there is no effective chord in $P$, i.e., $|\operatorname{SEC}(P)|=0$, we obtain $t\left(P+s_{1}+\ldots+s_{m}\right) \geq t(P)$. Here, note that if the sequence of chords is produced by Partition method $H$ then every chord has effect zero by Lemma 3 , because it is a primitive horizontal chord and exactly one endpoint is a vertex of $P$ by the assumption that $P$ has no effective chords. Thus, we 
have $t\left(P+s_{1}+\ldots+s_{m}\right)=t(P)$

We can implement Partition method $H$ in $O(n \log n)$ time by using the so-called plane sweep method, where $\mathrm{n}$ is the number of vertices of a polygonal region.

\section{Minimum Partition Problem}

We have shown that the minimum number of trapezoids needed to partition a polygonal region $P$ is given by the index of $P$, i.e., $t(P)=$ $n(P)+w(P)-h(P)-1$, if no effective chord (with positive effect) is available in $P$. Generally, we can use effective chords to decrease the number of trapezoids. To find the minimum number of trapezoids we must find the maximum number of effective chords available which are pairwise independent. The main objective here is to describe the problem (minimum partition problem) in a definite way. For this purpose we define a minimally effective chord and examine the relation between two such chords.

Definition 6. An effective chord $s$ is minimally effective if $s$ is primitive, or $s$ is non-primitive and no primtive subchord contained in $s$ is effective. For two minimally effective chords $s_{1}$ and $s_{2}$ of a polygonal region $P, s_{1}$ is independent of $s_{2}$ if $s_{1}$ is a minimally effective chord in $\mathrm{P}+\mathrm{s}_{2}$. (Clearly, in this case $\mathrm{s}_{2}$ is also independent of $\mathrm{s}_{1}$. Thus, $\mathrm{s}_{1}$ and $\mathrm{s}_{2}$ are often said to be independent.)

A minimally effective chord is characterized by the following lemmas, which are easily observed.

Lemma 6. A chord $\mathrm{s}$ of a polygonal region $\mathrm{P}$ is minimally effective if and only if the following (i) or (ii) holds.

(i) $s$ is a primitive horizontal chord joining two vertices of $P$.

(ii) $s$ is a non-horizontal chord joining two edges collinear with it.

Lemma 7. Let $\mathrm{s}$ be a minimally effective chord of a polygonal region P. Then, the effect of $\mathrm{s}$ in $\mathrm{P}$ is one, that is,

$\operatorname{effect}(s ; P)=t(P)-t(P+s)=1$.

Lemma 8. For each vertex $\mathrm{v}$ of a polygonal region $\mathrm{P}$, there are at most four minimally effective chords of $\mathrm{P}$ that contain $\mathrm{v}$ as an endpoint.

Lemma 9. Two minimally effective chords are independent if and only if the following (i) or (ii) holds.

(i) They do not intersect at all. 
(ii) They touch each other and one of the interior angles at their common point after drawing them is exactly $180^{\circ}$.

Fig. 7 illustrates three minimally effective chords $s(2-5-7)$ between vertices 2 and $7, s(3-5)$ between 3 and 5, and s(2-11) between 2 and 11 . The chord $s(3-5)$ touches $s(2-5-7)$ at the vertex 5 . The interior angle at vertex 5 is divided by the chords $s(3-5)$ and $s(2-5-7)$ into

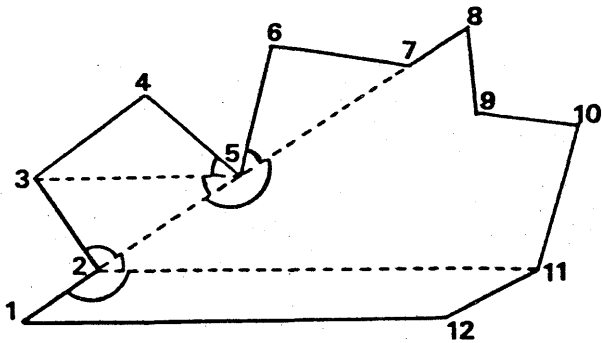

Fig. 7. Three minimally effective chords. Chords $s(2-5-7)$ and $s(3-5)$ are independent while chords $s(2-11)$ and $s(2-5-7)$ are not independent. four, that is, angle(2-5-3), angle(3-5-4), angle(6-5-7), and angle(2-5-7), where angle $(i-j-k)$ is the angle specified by the three vertices $i, j$ and $k$. Among them the angle(2-5-7) is exactly $180^{\circ}$. Therefore, the chords $s(2-5-7)$ and $s(3-5)$ are independent. On the other hand, the interior angle at vertex 2 is divided by chords $s(2-5-7)$ and $s(2-11)$ into three, any of which is not $180^{\circ}$. Therefore, the chord $s(2-5-7)$ is not independent of $\mathrm{s}(2-11)$.

Lemma 10. Let $s_{1}, s_{2}, \ldots, s_{D}$ be minimally effective chords of a polygonal region $\mathrm{P}$ which are pairwise independent. Then,

$t\left(P+s_{1}+s_{2}+\ldots+s_{D}\right)=t(P)-D$.

Proof. Immediate from Lemma 7 and Definition 6.D

Now it is evident that the maximum number of minimally effective chords which are pairwise independent coincides with the cardinality of a maximum independent set of a graph which is defined as follows.

Definition 7. An interesection graph of minimally effective chords of a polygonal region $P$, denoted by $G(P)$, is a graph whose node set $V(G(P))$ is the set of all minimally effective chords of $P$ and two nodes of $V(G(P))$ are joined by an arc if and only if they are not independent. For a set $\Phi$ of polygonal regions, $G(\Phi)$ is defined similarly.

A subset $\mathrm{N}$ of the node set of a graph $\mathrm{G}$ is called independent if there is no arc of $G$ joining any two nodes of $N$. A maximum independent set of a graph is an independent set of maximum cardinality. Dotted lines in Fig. 8 show the minimally effective chords of the polygonal region. The intersection graph of the minimally effective chords is shown in Fig. 9. Corresponding to Lemma 5, we have the following.

Lemma 11. Let $\mathrm{P}$ be a polygonal region and $\mathrm{s}$ be any chord of $\mathrm{P}$. Let 


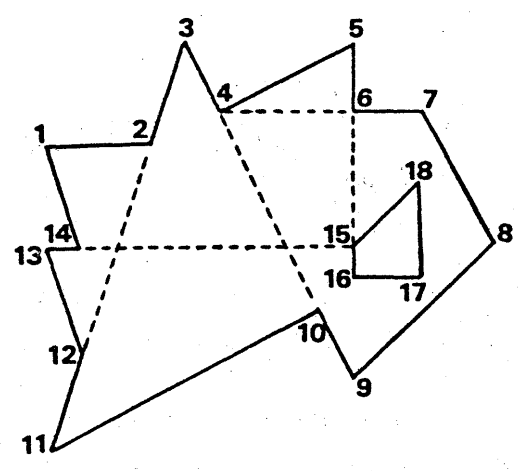

Fig. 8. Minimally effective chords of $P$ (dotted lines).

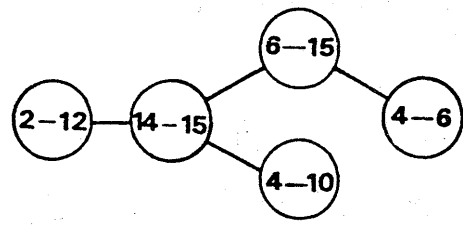

Fig. 9. Intersection graph $G(P)$ of minimally effective chords of $P$.

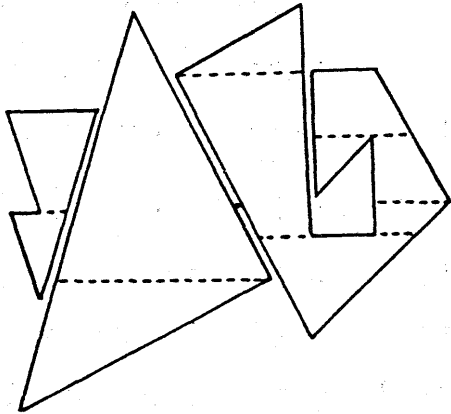

Fig. 10. Minimum partition of $P$ into trapezoids.

$d(P)(d(P+s)$, resp.) be the cardinality of a maximum independent set of the intersection graph $G(P)(G(P+s)$, resp.) of minimally effective chords of $P$ $(\mathrm{P}+\mathrm{s}$, resp. ). Then,

$d(P) \geq d(P+s)+$ effect $(s ; P)$.

Proof. By an argument similar to the one in the proof of Lemma $3 . \square$

Now, we can obtain an optimal partition method as follows:

[Partition method OPM]

Step 1. Find the set of minimally effective chords of a given polygonal region $P$.

Step 2. Find a maximum independent set $I *(P)$ of the intersection graph $G(P)$ of minimally effective chords of $P$.

Step 3. Using the chords corresponding to the nodes in $I *(P)$, partition $P$ into several polygonal regions $P_{1}, P_{2}, \ldots, P_{k}$.

Step 4. Partition each $P_{i}(i=1,2, \ldots, k)$ into trapezoids by Partition method $\mathrm{H}$.

Theorem 2. Partition method OPM partitions a polygonal region $P$ into a minimum number of trapezoids. The number $M *(P)$ of trapezoids in the minimum partition is given by $M *(P)=t(P)-d(P)$, that is,

$$
M *(P)=n(P)+w(P)-h(P)-d(P)-1,
$$

where $n(P), w(P)$, and $h(P)$ are the number of vertices, windows, and horizontal edges of $P$, respectively, $t(P)$ is the index of $P$, and $d(P)$ is the cardinality of a maximum independent set of the intersection graph $G(P)$ of minimally effective chords of $P$.

Proof. By Lemmas 2 and 10 and Theorem 1, Partition method OPM partitions the polygonal region $P$ into $t(P)-d(P)=n(P)+w(P)-h(P)-d(P)-1$ trapezoids. Thus, we have $M *(P) \leq t(P)-d(P)=n(P)+w(P)-h(P)-d(P)-1$. 
Next, we show

$M *(P) \geq t(P)-d(P)=n(P)+w(P)-h(P)-d(P)-1$.

Let $\mathrm{m}(\mathrm{P})$ be the minimum length of those sequences $\left(\mathrm{s}_{1}, \mathrm{~s}_{2}, \ldots, \mathrm{s}_{\mathrm{m}(\mathrm{P})}\right)$ of the chords which partition a polygonal region $\mathrm{P}$ into a minimum number of trapezoids. Lemma 1 assures that there is an upper bound for $m(P)$, such as $t(P)+1 \geq M^{*}(P)+1 \geq m(P)$. Thus, $m(P)$ is the well defined number for every polygonal region $P$. We shall show (3.2) by induction on $m(P)$. If $m(P)=0$ then $P$ is a trapezoid and (3.2) trivially holds. Assume that (3.2) is true for all polygonal regions $\mathrm{P}$ with $\mathrm{m}(\mathrm{P})<\mathrm{m}(\mathrm{m} \geq 1)$. Let $\mathrm{P}$ be a polygonal region with $\mathrm{m}(\mathrm{P})=\mathrm{m}$. Let $\left(\mathrm{s}_{1}, \mathrm{~s}_{2}, \ldots, \mathrm{s}_{\mathrm{m}}\right)$ be a sequence of chords which partitions $P$ into a minimum number of trapezoids. Set $s=s_{1}$. We first consider the case where $\mathrm{P}+\mathrm{s}$ has exactly one polygonal region. Then, $\left(s_{2}, \ldots, s_{m}\right)$ is a sequence of chords which partitions $\mathrm{P}+\mathrm{s}$ into a minimum number of trapezoids. Since $\mathrm{m}(\mathrm{P}+\mathrm{s})<\mathrm{m}$, we have by the inductive hypothesis, $\mathrm{M} *(\mathrm{P})=\mathrm{M} *(\mathrm{P}+\mathrm{s}) \geq \mathrm{t}(\mathrm{P}+\mathrm{s})-\mathrm{d}(\mathrm{P}+\mathrm{s})=\mathrm{n}(\mathrm{P}+\mathrm{s})+\mathrm{w}(\mathrm{P}+\mathrm{s})-\mathrm{h}(\mathrm{P}+\mathrm{s})-\mathrm{d}(\mathrm{P}+\mathrm{s})-1$. Thus, by Lemma 11 , we have.

$M *(P)=M *(P+s) \geq t(P+s)-d(P+s)=t(P)-e f f e c t(s ; P)-d(P+s) \geq t(P)-d(P)$. (3.3) Even if $P+s$ consists of $k(k \geq 2)$ polygonal regions $P_{1}, \ldots, P_{k}$, we can apply the above argument to each $\mathrm{P}_{i}$ and obtain (3.3), where $\mathrm{M} *(\mathrm{P}+\mathrm{s})$ is the sum of all $\mathrm{M} *\left(\mathrm{P}_{i}\right)$. Thus, we obtain (3.2) for all polygonal regions $\mathrm{P}$, and the theorem is proved.

A maximum independent set of the intersection graph $G(P)$ shown in Fig. 9 is $\{2-12,6-15,4-10\}$, where each chord is represented by a couple of its endpoints. Fig. 10 illustrates the trapezoids produced by Partition method OPM.

Steps 1, 3, and 4 in Partition method OPM can be easily implemented in $O\left(n^{2}\right)$ time. Thus, the efficiency of Partition method OPM can be estimated by Step 2. An intersection graph of minimally effective chords of a polygonal region is a rather restricted graph. However, it is not easy to find a maximum independent set of the intersection graph because we have the following theorem. To describe the theorem, we need some terms.

Definition 8. An intersection graph of a class A of sets is a graph whose node set is the class $A$ and two nodes are joined by an arc if and only if the corresponding two sets have an element in common. An intersection graph of a set of straight lines in the plane (chords of a circle, curves in the plane, resp.) is a straight-lines-in-the-plane graph (circle graph, curves-in-the-plane graph, resp.). 


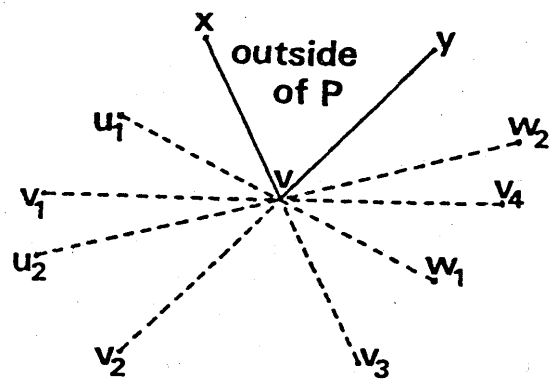

(a)

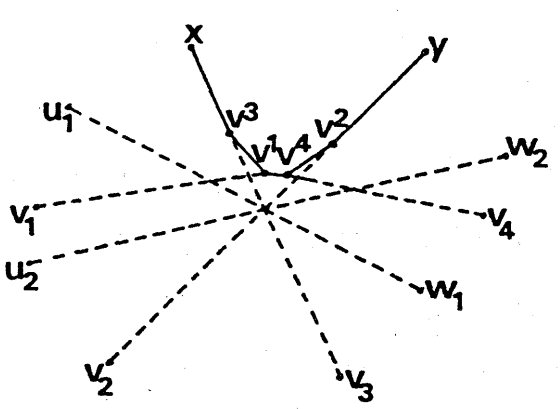

(b)

Fig. 11. Transformation of a polygonal region $P$.

Theorem 3. The class of intersection graphs of minimally effective chords of polygonal regions is the class of straight-1ines-in-the-plane graphs.

Proof. We first show that the class of intersection graphs of minimally effective chords of polygonal regions is a subset of the class of straight-lines-in-the-plane graphs.

Let $\mathrm{P}$ be a polygonal region and $\mathrm{L}$ be the set of minimally effective chords of $P$. We assume that $L$ satisfies the following condition (X):

(X) Any chord in $\mathrm{L}$ is primitive and no two chords in $\mathrm{L}$ have an endpoint in common.

Even if $L$ does not satisfy the condition ( $X), L$ can always be modified to satisfy the condition (X). For example, let $\mathrm{V}$ be a common endpoint of four chords $\left(v, v_{1}\right),\left(v, v_{2}\right),\left(v, v_{3}\right)$, and $\left(v, v_{4}\right)$ in L. Also let chords $\left(\mathrm{u}_{1}, \mathrm{w}_{1}\right)$ and $\left(\mathrm{u}_{2}, \mathrm{w}_{2}\right)$ in $\mathrm{L}$ touch $\mathrm{v}$ as shown in Fig 11(a). Then, consider four points $\mathrm{v}^{1}, \mathrm{v}^{2}, \mathrm{v}^{3}$ and $\mathrm{v}^{4}$ sufficiently close to $\mathrm{v}$ and replace $\mathrm{v}$ with these four points and $\left(\mathrm{v}, \mathrm{v}_{1}\right),\left(\mathrm{v}, \mathrm{v}_{2}\right),\left(\mathrm{v}, \mathrm{v}_{3}\right),\left(\mathrm{v}, \mathrm{v}_{4}\right)$ with $\left(\mathrm{v}^{1}, \mathrm{v}_{1}\right),\left(\mathrm{v}^{2}, \mathrm{v}_{2}\right)$, $\left(\mathrm{v}^{3}, \mathrm{v}_{3}\right),\left(\mathrm{v}^{4}, \mathrm{v}_{4}\right)$ as shown in Fig. $11(\mathrm{~b})$. Although $\left(\mathrm{v}^{1}, \mathrm{v}_{1}\right)$ and $\left(\mathrm{v}^{4}, \mathrm{v}_{4}\right)$ are no more effective chords of the resulting polygonal region, it can be easily seen that the intersection graph of the set of the resulting chords coincides with $G(P)$ by the finiteness of edges of $P$. Other cases can be similarly reduced. Thus, any intersection graph of minimally effective chords of a polygonal region is a straight-lines-in-the-plane graph.

Next, we show that the class of straight-lines-in-the-plane graphs is a subset of the class of intersection graphs of minimally effective chords of polygonal regions, which will be shown below.

Let $G$ be a straight-lines-in-the-plane graph, and $L$ be a representation of $\mathrm{G}$, that is, a set of straight lines in the plane which realizes the graph G. By the finiteness of $\mathrm{L}$, we can assume that all 


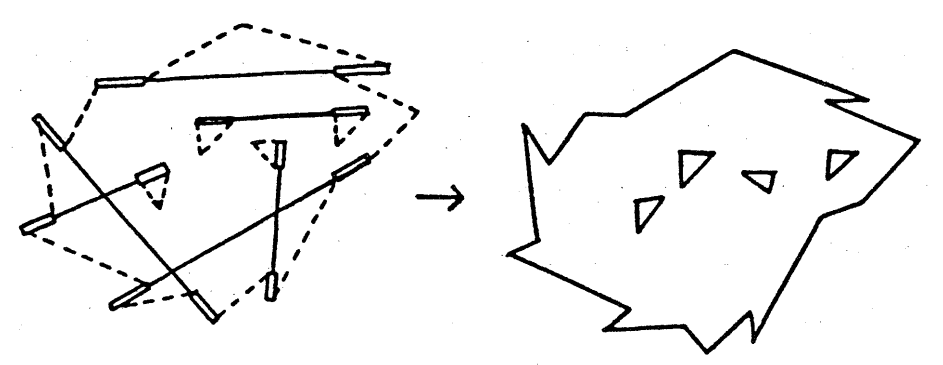

Fig. 12. Straight lines in the plane and the associated polygonal region.

endpoints of the lines are distinct and that any two lines are not collinear. Then, we can make a polygonal region $P$ whose intersection graph of minimally effective chords coincides with the given graph $G$ as follows. We first find the convex hull $\mathrm{C}(\mathrm{L})=\mathrm{v}_{1}, \mathrm{v}_{2}, \ldots, \mathrm{v}_{\mathrm{m}+1}=\mathrm{v}_{1}$ for the set of all endpoints of the lines of $\mathrm{L}$. Then, we extend each line of L sufficiently short at both endpoints. By the finiteness of $\mathrm{L}$, we can assume that the set of the resulting lines also represents $G$ and that any two endpoints do not have the same y-coordinate. We then modify the convex hull $\mathrm{C}(\mathrm{L})$ and obtain a polygon as illustrated in Fig. 12. For each endpoint inside the polygon we assign a small triangle appropriately. Let $P$ be the resulting polygonal region. Then, it is easy to show that the intersection graph $G(P)$ of minimally effective chords of $P$ coincides with $G$. $\square$

A straight-1ines-in-the-plane graph is a curves-in-the-plane graph, and a circle graph is a straight-1ines-in-the-plane graph [5,14]. The maximum independent set problem is solvable in polynomial time for circle graphs $[7,11]$, while it is NP-complete for curves-in-the-plane graphs, because planar graphs are curves-in-the-plane graphs [5] and the maximum independent set problem is NP-complete for planar graphs [6]. Recently, Kashiwabara [15] showed that the maximum independent set problem for straight-lines-in-the-plane graphs is NP-complete. He proved that the planar 3-satisfiability problem, which is known to be NP-complete [17], is polynomially transformable to the maximum independent set problem for straight-lines-in-the-plane graphs. The maximum independent set problem for straight-lines-in-the-plane graphs is polynomially transformable to the problem of minimum partition of polygonal regions into trapezoids, which is obtained by the same argument in the proof of Theorem 3. Thus, we have the following theorem.

Theorem 4. The problem of partitioning a polygonal region into a minimum number of trapezoids is NP-complete. 
4. Exact Algorithms for Polygonal Regions With a Fixed Number of Windows

In the preceding section we showed that it is NP-complete to partition a polygonal region into a minimum number of trapezoids. However, for a polygonal region with a fixed number of windows, a polynomial-time algorithm may be possible. In this section we first present an $O\left(n^{2}\right)$ time algorithm for partitioning a polygonal region without windows into a minimum number of trapezoids. Next, we describe a polynomial-time algorithm for a polygonal region with a fixed number of windows.

Our $O\left(\mathrm{n}^{2}\right)$ time algorithm is based on two results: one is our efficient algorithm for finding a maximum independent set of a circle graph [11], which will be described later; and the other is the following theorem.

Theorem 5: The class of intersection graphs of minimally effective chords of polygonal regions without windows is the class of circle graphs.

Proof. We first show that the class of intersection graphs of minimally effective chords of polygonal regions without windows is a subset of the class of circle graphs.

Let $\mathrm{P}=\mathrm{v}_{1}, \mathrm{v}_{2}, \ldots, \mathrm{v}_{\mathrm{n}}, \mathrm{v}_{1}$ be a polygonal region without windows. Let $\mathrm{L}$ be the set of minimally effective chords of $\mathrm{P}$. By the same reason as in the proof of Theorem 3, we can assume that $\mathrm{L}$ satisfies the condition ( $\mathrm{X})$ : Any chord in $\mathrm{L}$ is primitive and no two chords in $\mathrm{L}$ have an endpoint in common. Thus, by Lemma 9, two minimally effective chords of $P$ are independent if and only

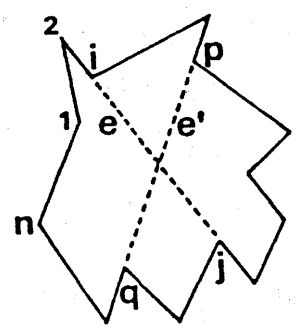

Fig. 13. Two non-independent chords of $P$. if they do not intersect at all. Since $\mathrm{P}$ is a polygon, for any chords $e=\left(v_{i}, v_{j}\right)$ and $e^{\prime}=\left(v_{p}, v_{q}\right)$ in $L(1 \leq i<j \leq n, 1 \leq p<q \leq n)$, e and $e^{\prime}$ intersect if and only if one of $\left\{\mathrm{v}_{p}, \mathrm{v}_{q}\right\}$ lies on the $\operatorname{arc} \mathrm{v}_{i}, \mathrm{v}_{i+1}, \ldots, \mathrm{v}_{j}$ of $\mathrm{P}$ and the other on the $\operatorname{arc} v_{j}, v_{j+1}, \ldots, v_{n}, v_{1}, \ldots, v_{i}$, that is, $i<p<j<q$ or $p<i<q<$ $j$ (Fig. 13). Thus, if we consider that chords of a circle $C$ are obtained in a way as shown in Fig. 14 corresponding to the minimally effective chords of $P$, then the intersection graph of the chords of $C$ coincides with $G(P)$, the intersection graph of minimally effective chords of $P$. This implies that $G(P)$ is a circle graph.

Conversely, by an argument similar to the one in the proof of Theorem 3 , we can obtain that any circle graph is realized by an intersection graph of minimally effective chords of a polygonal region without windows.

Since Steps 1, 3, and 4 in the optimal Partition method OPM in Section 
3 can be easily implemented in $O\left(n^{2}\right)$ time, if step 2 can be implemented in $o\left(n^{2}\right)$ time for a polygonal region $P$ without windows, then the Partition method OPM requires only $0\left(n^{2}\right)$ time. Note that even if the set $L$ of minimally effective chords of $P$ does not satisfy

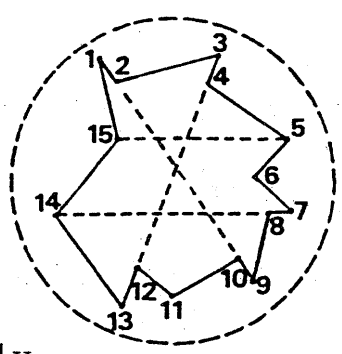

(a)

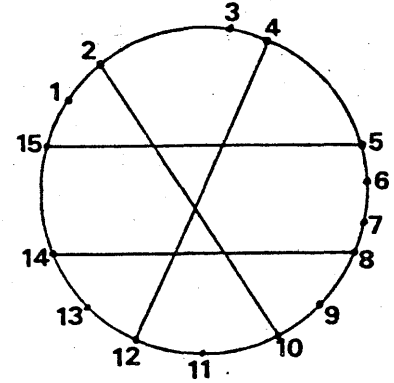

(b) the condition (X) stated above, L can always be modified to satisfy the condition $(X)$ in $O\left(n^{2}\right)$ time. Since $G(P)$ is a circle graph by Theorem 5, we will try to design an $O\left(n^{2}\right)$ time algorithm

Fig. 14. Circular representation of a polygonal region $P$ without windows.

(a) Minimally effective chords of a polygonal region $P$. (b) chords of a circle. for finding a maximum independent set of a circle graph. We first present an $O(n \log n)$ time algorithm for finding a maximum-weight independent set of an interval graph. Then, using this algorithm for an interval graph, we describe an $\mathrm{O}\left(\mathrm{n}^{2}\right)$ time algorithm for finding a maximum independent set of a circle graph. Here, interval graph is an intersection graph of a set of intervals and a maximum-weight independent set is a independent set of a maximum weight with respect to a given weight function on the node set.

Consider an interval graph $G_{n}$ whose intevals are given by $I_{i}=\left[x_{i}{ }^{-}, x_{i}{ }^{+}\right]$ $(i=1,2, \ldots, n)$. Without loss of generality, we can assume that $2 n$ numbers $\mathrm{x}_{i}{ }^{-}, \mathrm{x}_{i}{ }^{+}$are distinct and $\mathrm{x}_{1}{ }^{-}<\mathrm{x}_{2}{ }^{-}<\ldots<\mathrm{x}_{\mathrm{n}}{ }^{-}$. To each interval $\mathrm{I}_{i}$, a positive weight $w(i)$ is attached. For this interval graph $G_{n}$, a maximumweight independent set can be found by the algorithm shown in Fig. 15 which uses a kind of the so-called plane sweep method, where we consider a dummy interval $I_{0}=[-\infty,-\infty]$. For $i=1,2, \ldots, n$, let $G_{i}$ be the interval graph of intervals $I_{1}, I_{2}, \ldots, I_{i}$. Then $S_{i}$ is a maximum-weight independent set of $G_{i}$ that contains the interval $I_{i}$ if and only if $S_{i}-\left\{I_{i}\right\}$ is a maximum-weight independent set of the interval graph of those intervals $I_{m}$ with $x_{m}{ }^{+}<x_{i}{ }^{-}$. Thus we can easily obtain the following observation by induction on $k$, which will help us to understand the algorithm well:

Let $\mathrm{x}$ be the $\mathrm{k}$-th number and $\mathrm{J}$ be a maximum-weight independent set of the interval graph of those intervals $\mathrm{I}_{\mathrm{m}}$ with $\mathrm{x}_{\mathrm{m}}{ }^{+}<\mathrm{x}$. Then, just before the $k$-th iteration, $U$ is the weight of $J$ and, for each $i$ with $x_{i}{ }^{-}<x, W(i)$ is the weight of a maximum-weight independent set of $G_{i}$ that contains $I_{i}$. Furthermore, $j$ indicates the rightmost interval contained in $\mathrm{J}$, that is, $\mathrm{x}_{\mathrm{j}}{ }^{+}<\mathrm{x}_{\mathrm{m}}{ }^{+}$for any interval $\mathrm{I}_{\mathrm{m}}$ in $\mathrm{J}$ if $\mathrm{J} \neq \phi, j=0$ otherwise. 
Procedure MWIS;

begin

sort $2 \mathrm{n}$ numbers $\mathrm{x}_{i}^{-}, \mathrm{x}_{i}^{+}(i=1,2, \ldots, \mathrm{n})$ in increasing order; $\mathrm{j}:=0 ; \mathrm{U}:=0$;

for $k:=1$ to $2 n$ do

begin

if the $\mathrm{k}$-th number is $\mathrm{x}_{i}{ }^{-}$then

begin

comment: $U$ is the weight of a maximum-weight independent set of an interval graph of intervals $I_{m}$ with $x_{m}^{+}<x_{i}^{-}$ $W(i):=U+W(i) ; \quad \stackrel{m}{p}(i)=j$

if (the $k-t h$ number is $x_{i}^{+}$) and $(U<W(i))$ then
begin $U:=W(i) ; \quad$ end

end; begin $U:=W(i) ; \quad j:=i$ end

find $i$ such that $W(i)=\max \{W(j) \mid j=1,2, \ldots, n\} ; S:=\phi$;

while $i \neq 0$ do

begin $S:=S \cup\{i\} ; \quad i:=p(i)$ end;

end;

comment: $\mathrm{S}$ is a maximum-weight independent set

Fig. 15. A maximum-weight independent set algorithm for an interval graph $G_{n}$.

Thus, we have the following lemma.

Lemma 12. For $i=1,2, \ldots, n$, consider an interval graph $G_{i}$ consisting of $I_{1}, I_{2}, \ldots, I_{i}$. Then, $W(i)$ is the weight of a maximum-weight independent set $S_{i}$ of $G_{i}$ that contains $I_{i}$, and $p(i)$ indicates the rightmost interval contained in $S_{i}-\left\{I_{i}\right\}$, i.e.,

$\mathrm{x}_{\mathrm{p}(\mathrm{i})}^{+}=\max \left\{\mathrm{x}_{\mathrm{h}}^{+} \mid \mathrm{I}_{\mathrm{h}} \in\left(\mathrm{S}_{\mathrm{i}} \cup\left\{\mathrm{I}_{0}\right\}\right)-\left\{\mathrm{I}_{i}\right\}\right\}$.

This algorithm takes $O(n \log n)$ time in sorting $2 n$ numbers $x_{i}^{-}, x_{i}^{+}$ $(i=1,2, \ldots, n)$, and $O(n)$ time in the other parts, hence we have the following.

Lemma 13. A maximum-weight independent set of an interval graph composed of $n$ intervals can be found in $O(n \log n)$ time. Furthermore, if $2 n$ endpoints of intervals are given in sorted order, it can be found in $O(n)$ time.

Gavril [6] has shown that a maximum-weight independent set of a circle graph can be found by solving $n$ times the problem of finding a maximumweight independent set of an interval graph consisting of at most $n$ intervals. He has considered that the problem for interval graphs can be solved in $O\left(\mathrm{n}^{2}\right)$ time, and that the total time complexity of his algorithm is $O\left(n^{3}\right)$. In solving $n$ problems for those interval graphs, we need sorting only once in total, so that, by Lemma 13, we can obtain the following: 
Theorem 6. A maximum-weight independent set of a circle graph composed of $n$ chords of a circle can be found in $0\left(n^{2}\right)$ time.

By Theorems 5 and 6 we have the following main theorem in this section.

Theorem 7. Partition method OPM can be implemented in $O\left(\mathrm{n}^{2}\right)$ time for a polygonal region $\mathrm{P}$ without windows, where $\mathrm{n}$ is the number of vertices of P.

Next, we present an $0\left(\mathrm{n}^{2+\mathrm{w}}\right)$ time algorithm for partitioning a polygonal region with $\mathrm{w}$ windows into a minimum number of trapezoids after giving a few preliminaries.

Let $\mathrm{P}$ be a polygonal region with $\mathrm{w}$ windows $\mathrm{W}_{1}, \mathrm{~W}_{2}, \ldots, \mathrm{W}_{\mathrm{w}}$. We consider the external polygon of $\mathrm{P}$ to be a window $\mathrm{W}_{0}$. For a minimally effective chord $s, s$ is called a connector of $P$ if it connects two distinct windows (Fig. 3 (c) and (d)). If a minimally effective chord s connects two points on the same window (Fig. 3 (a) and (b)), then it is called a proper chord of $P$. For two proper chords $s$ and $s '$ such that $s$ connects two points on some window and $s^{\prime}$ connects two points on another window, $\mathrm{S}$ and $S^{\prime}$ are clearly independent. By an argument similar to the one in the proof of Theorem 5, we have that the intersection graph $G_{\left(W_{i}\right)}$ of those minimally effective chords of $\mathrm{P}$ which are proper chords connecting two points on $W_{i}$ is a circle graph. Thus we have the following lemma.

Lemma 14. For a polygonal region $\mathrm{P}$ with $\mathrm{w}$ windows $\mathrm{W}_{1}, \mathrm{~W}_{2}, \ldots, \mathrm{W}_{\mathrm{w}}$ and the external polygon $W_{0}$, the intersection graph $G_{0}(P)$ of those minimally effective chords of $\mathrm{P}$ which are proper is a disjoint union of circle graphs $G\left(W_{i}\right)(i=0,1, \ldots, w)$, where $G\left(W_{i}\right)$ is the intersection graph of those minimally effective chords of $\mathrm{P}$ which are proper chords connecting two points on $W_{i}$.

By Lemma 14, we can obtain the following partition method for a polygonal region with $\mathrm{w}$ windows.

[Partition method B for a polygonal region with w windows]

Step 1. For a given polygonal region with w windows, find the set of minimally effective chords of $\mathrm{P}$.

Step 2. Find a maximum independent set $I *(P)$ of the intersection graph $\mathrm{G}(\mathrm{P})$ of minimally effective chords of $\mathrm{P}$ as follows.

Step 2.1. Find a maximum independent set $I_{0}$ of the intersection graph $G_{0}(P)$ of those minimally effective chords of $P$ which are proper. 
Step 2.2. For each connector $s_{j}(j=1,2, \ldots, m)$, find a maximum independent set $\mathrm{I} *\left(\mathrm{P}+\mathrm{s}_{j}\right)$ of the intersection graph $\mathrm{G}\left(\mathrm{P}+\mathrm{s}_{j}\right)$ of minimally effective chords of the polygonal region $\mathrm{P} \mathrm{s}_{j}$ and set $I_{j}=I *\left(P+s_{j}\right) \cup\left\{s_{j}\right\}$.

Step 2.3. Find a set $I *(P)$ of maximum cardinality among the sets $I_{0}, I_{1}, \ldots, I_{m}$.

Step 3. Using the minimally effective chords corresponding to the nodes in $I *(P)$, partition $P$ into several polygonal regions $P_{1}, P_{2}, \ldots, P_{k}$. Step 4. Partition each $\mathrm{P}_{i}(i=1,2, \ldots, k)$ into trapezoids by Partition method $\mathrm{H}$.

Theorem 8. Partition method B correctly partitions a polygonal region $\mathrm{P}$ with $\mathrm{w}$ windows into a minimum number of trapezoids and can be implemented in $O\left(n^{2+w}\right)$ time, where $n$ is the number of vertices of $P$.

Proof. Since every maximum independent set of the intersection graph $G(P)$ either contains connector $s_{j}$ or not, a maximum independent set of $G(P)$ is correctly obtained in Step 2. Thus, the correctness of Partition method B follows from Theorem 2.

Steps 1, 3, and 4 in Partition method $B$ can be implemented in $O\left(n^{2}\right)$ time. Thus we consider only Step 2. Let $\mathrm{m}$ be the number of those minimally effective chords which are connectors of $P$. We shall show by induction on $\mathrm{m}$ and $\mathrm{w}$ that the time complexity $\mathrm{T}(\mathrm{n} ; \mathrm{m}, \mathrm{w})$ required in Step 2 is estimated as follows:

$$
\mathrm{T}(\mathrm{n} ; \mathrm{m}, \mathrm{w})=\mathrm{O}\left(\mathrm{m}^{\min (\mathrm{m}, \mathrm{w})} \mathrm{n}^{2}\right) \text {. }
$$

If $\mathrm{m}=0$, then (4.1) holds for all $\mathrm{w} \geq 0$ by Theorem 6 and Lemma 14. Assume that $(4.1)$ holds for all $\mathrm{w} \geq 0$ and all $\mathrm{m}<\mathrm{p}$. Now, let $\mathrm{P}$ be a polygonal region with $w$ windows and $p$ connectors $(w \geq 0, p>0)$. Step 2.1 requires $o\left(\mathrm{n}^{2}\right)$ time by Theorem 6 and Lemma 14. Step 2.2 is divided into two parts: one is to find the minimally effective chords of $\mathrm{P}_{\mathrm{s}} \mathrm{s}_{j}(j=1,2, \ldots, \mathrm{p})$, which requires $O(n)$ for each $j$; the other is to find a maximum independent set $I *\left(P+s_{j}\right)$ of $G\left(P+s_{j}\right)$, which requires $T\left(n_{j} ; m_{j}, w_{j}\right)$ for each $j$, where $n_{j}, m_{j}$ and $\mathrm{w}_{j}$ are the number of vertices, connectors and windows of $\mathrm{P}+\mathrm{s}_{j}$, respectively. Thus Step 2.2 requires

$$
\sum_{j=1}^{p}\left(T\left(n_{j} ; m_{j}, w_{j}\right)+O(n)\right)
$$

time. Since $m_{j}<p-1$ and $w_{j}<w-1$ for each $j(j=1,2, \ldots, p)$, we have by the inductive hypothesis that Step 2.2 requires

$$
O\left(p \cdot p^{\min (p-1, w-1)} n^{2}\right)
$$

time. Step 2.3 requires $O(p+n)$ time. Thus Step 2 is shown to require only $\mathrm{o}\left(\mathrm{p}^{\min (\mathrm{p}, \mathrm{w})} \mathrm{n}^{2}\right)$ time and $(4.1)$ is proved for all $\mathrm{m}$ and $\mathrm{w}$. 
Since $m \leq n$, we have the theorem.

Finally, we give a remark on a heuristic method of finding a maximum independent set of the intersection graph of minimally effective chords. Let $\mathrm{P}$ be a polygonal region

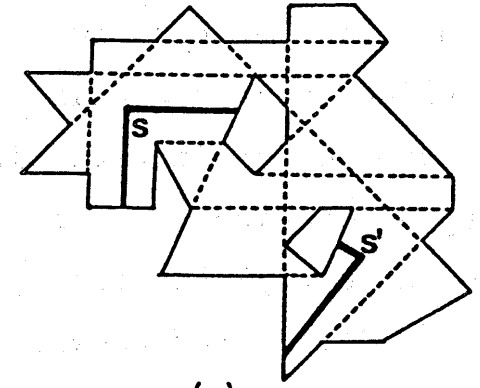

(a)

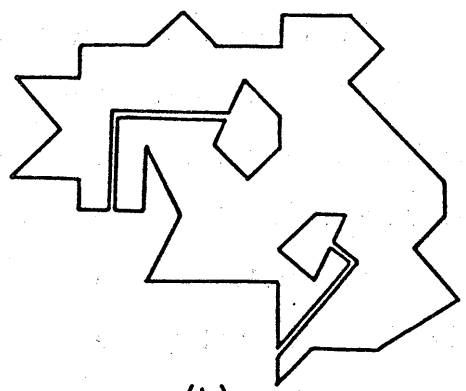

(b) with $\mathrm{w}$ windows $\mathrm{W}_{1}, \mathrm{w}_{2}, \ldots, \mathrm{w}_{\mathrm{w}}$. Consider the external polygon to be a window $W_{0}$. Let $F(P)$ be Fig. 16. (a) Graph $F(P)$ of a polygonal region $P$ and two broken lines $s$ and $s^{\prime}$. (b) Polygonal region obtained by drawing two broken lines $s$ and $s^{\prime}$. the plane graph obtained from $P$ by drawing al1 minimally effective chords of P (Fig. 16(a)). A node of $F(P)$ is a vertex of $P$ or an intersection point of some minimally effective chords of $P$. Clearly, $F(P)$ has $O\left(n^{2}\right)$ nodes. If $F(P)$ has a region $f$ adjacent to two distinct windows $\mathrm{w}_{i}$ and $\mathrm{w}_{j}$ of $\mathrm{P}$, then we can merge two windows into one by drawing broken line $s$ inside the region $f$ connecting a point on $W_{i}$ and a point on $W_{j}$. As far as finding a maximum independent set of $G(P)$ we can draw such lines, because the intersection graph is not changed. Thus, this may be applied to decrease the number of windows of $P$ in Step 2 of Partition method B (see Fig. 16(b) and Theorem 8).

\section{Approximation Algorithms}

In the preceding section, we have presented an $0\left(n^{2+w}\right)$ time algorithm for partitioning a polygonal region with w windows into a minimum number of trapezoids, although, in general, this problem for polygonal regions with the unbounded number of windows is NP-complete. However, this algorithm requires too much time to be said practical. From the viewpoint of practical applications, a minimum partition is not so important. A near optimal partition is quite satisfactory in practice.

In this section, we present an approximation method for this problem. As a special case of this method, we can obtain an $O(n$ log $n$ ) time approximation algorithm with absolute performance ratio 3 . Note that for an approximation algorithm C for a minimization (maximization, resp.) problem, the absolute performance ratio $\mathrm{R}(\mathrm{C})$ of the algorithm $\mathrm{C}$ is defined by

$$
R(C)=\sup \{|C(I)| /|O P T(I)|\} \quad(R(C)=\inf \{|C(I)| /|O P T(I)|\}, \operatorname{resp} .),
$$


where OPT(I) is an optimal solution and $\mathrm{C}(\mathrm{I})$ is an approximation solution obtained by the algorithm $\mathrm{C}$ for an instance $\mathrm{I}$ of the problem. Our approximation method is based on an approximation method AMIS for the maximum independent set problem for intersection graphs of minimally effective chords of polygonal regions. If the absolute performance ratio of the method AMIS is $1-1 / c \quad(c \geq 1)$ then the ratio of our method is given by $1+2 / \mathrm{c}$ for polygonal regions $(1+1 / \mathrm{c}$ for polygonal regions with zero or one window).

\section{[Partition method A]}

Step 1. Find the set of minimally effective chords of a given polygonal region $P$.

Step 2. Find an independent set $I(P ; A)$ of the intersection graph $G(P)$ of the minimally effective chords by an approximation method AMIS with the absolute performance ratio $1-1 / \mathrm{c}$.

Step 3. Using the chords corresponding to the nodes in $\mathrm{I}(\mathrm{P} ; \mathrm{A})$, partition $\mathrm{P}$ into several polygonal regions $\mathrm{P}_{1}, \mathrm{P}_{2}, \ldots, \mathrm{P}_{\mathrm{k}}$.

Step 4. Partition each $\mathrm{P}_{i}(i=1,2, \ldots, k)$ into trapezoids by Partition method H.

Theorem 9. If the absolute performance ratio of the approximation method AMIS is $(1-1 / c)$ for the maximum independent set problem on intersection graphs of minimally effective chords of polygonal regions, then the absolute performance ratio of Partition method $A$ is $(1+2 / c)$ for general polygonal regions and $(1+1 / c)$ for polygonal regions with zero or one window.

Proof. Let $I(P ; A)$ be an independent set of $G(P)$ obtained by the method AMIS and $I *(P)$ be a maximum independent set of $G(P)$. Let $M(P ; A)$ be the number of trapezoids obtained by Partition method $A$. There may be an independent set containing all nodes in $I(P ; A)$ and other nodes of $G(P)$ which correspond to horizontal chords. Thus, by Lemmas 2 and 10 , we have

$$
M(P ; A) \leq n(P)+w(P)-h(P)-|I(P ; A)|-1 .
$$

By Theorem 2, we have

$$
M *(P)=n(P)+w(P)-h(P)-|I *(P)|-1 \text {. }
$$

We shall prove $(\mathrm{c}+2) \mathrm{M} *(\mathrm{P})-\mathrm{cM}(\mathrm{P} ; \mathrm{A})>0$ (i.e., $\mathrm{M}(\mathrm{P} ; \mathrm{A}) / \mathrm{M} *(\mathrm{P})<1+2 / \mathrm{c}$ ) via Lemma 1 .

Suppose that we applied the optimal partition method OPM to the given polygonal region $\mathrm{P}$ and obtained the set $\mathrm{D}^{\prime}$ of minimally effective chords corresponding to a maximum independent set $I *(P)$ of the intersection graph $G(P)$. Let $D^{\prime \prime}$ be the set of chords drawn in Step 4 of the optimal partition 
method OPM. Then, by Lemma 1 , we have the following inequality

$$
M *(P) \geq\left|D^{\prime}\right|+\left|D^{\prime \prime}\right|-w(P)+1 \text {. }
$$

The equality holds when every chord in $D^{\prime}$ and $D^{\prime \prime}$ is primitive. Since

$$
D^{\prime}=|I *(P)| \text {, by }(5.2) \text { and }(5.3) \text {, we have }
$$

$$
\begin{aligned}
& 2|I *(P)| \leq n(P)+2 w(P)-h(P)-2, \text { and } \\
& M *(P)+w(P)+1-|I *(P)| \geq 2 .
\end{aligned}
$$

Thus we have

$$
\begin{aligned}
|I *(P)| & \leq(3 / 2)(n(P)+2 w(P)-h(P)-2)-2|I *(P)| \\
& <2(n(P)+w(P)-h(P)-|I *(P)|-1)=2 M *(P),
\end{aligned}
$$

because it is easily seen that any window contains at least two non-horizontal polygon edges and $n(P)-h(P)-2 w(P) \geq 0$. From the assumption, we have $|I(P ; A)| /|I *(P)| \geq 1-1 / c$. Thus, by (5.1) and (5.5), we have

$$
\begin{aligned}
(\mathrm{c}+2) \mathrm{M} *(\mathrm{P})-\mathrm{cM}(\mathrm{P} ; \mathrm{A}) & \geq \mathrm{c}(|\mathrm{I}(\mathrm{P} ; \mathrm{A})|-|\mathrm{I} *(\mathrm{P})|)+2 \mathrm{M} *(\mathrm{P}) \\
& >\mathrm{c}|\mathrm{I}(\mathrm{P} ; \mathrm{A})|-(\mathrm{c}-1)|\mathrm{I} *(\mathrm{P})| \geq 0 .
\end{aligned}
$$

If $w(P)=0$ or 1 , then by $(5.1)$ and $(5.4)$ we have

$(\mathrm{c}+1) \mathrm{M} *(\mathrm{P})-\mathrm{cM}(\mathrm{P} ; \mathrm{A}) \geq 0$.

If we set $c=1$, then we have the following theorem for the $O(n \log n)$ time Patition method $\mathrm{H}$.

Theorem 10. The absolute performance ratio $\mathrm{R}(\mathrm{H})$ of Partition method $\mathrm{H}$ is 3 ( 2 , resp.) for the problem of the minimum partition of polygonal regions (polygonal regions without windows, resp.) into trapezoids.

Note that the bounds are best possible. For the polygonal region $\mathrm{P}$ without windows shown in Fig. 17, $M(P ; H)=2 m+1$ and $M *(P)=m+1$. Thus we have

$$
\mathrm{M}(\mathrm{P} ; \mathrm{H}) / \mathrm{M} *(\mathrm{P})=2-1 /(\mathrm{m}+1) .
$$

The ratio is asymptotically two. On the other hand, the polygonal region $\mathrm{P}$ shown in Fig. 18 contains $m$ windows. We can easily see that $M(P ; H)=3 m+5$ and $M *(P)=m+3$. Thus,

$$
\mathrm{M}(\mathrm{P} ; \mathrm{H}) / \mathrm{M} *(\mathrm{P})=3-4 /(\mathrm{m}+3) \text {. }
$$

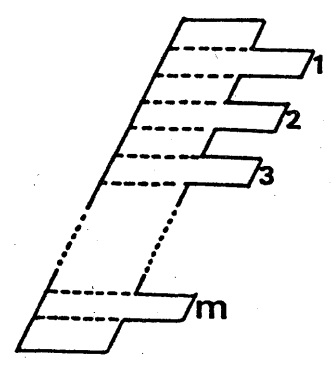

Fig. 17. The worst case polygonal region $P$ without windows.

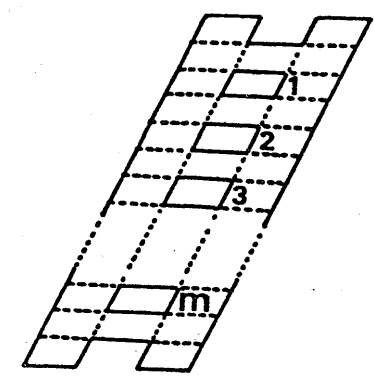

Fig. 18. The worst case polygonal region $P$ with windows.

We have known that if we have an approximation algorithm with the absolute performance ratio bounded by some constant for the maximum independent set problem on the intersection graphs then we can design an approximation algorithm for the minimum partition problem of polygonal regions whose absolute performance ratio is better than that of Partition 
method H. Unfortunately, we know no polynomial-time approximation algorithm with the absolute performance ratio bounded by some constant for the maximimum independent set problem of general graphs [6]. It might be true for straight-lines-in-the-plane graphs, although no proof has been done yet. Thus, if we consider all possible straight-lines-in-the-plane graphs then there may be little hope to obtain a $(1-1 / c)$ approximation algorithm for the maximum independent set problem for some $c>1$.

From the practical point of view, we do not need to consider all possible intersection graphs. For example, VLSI artwork data contains $\mathrm{k} \cdot 45^{\circ}$ $(k=0,1,2,3)$ lines to about 90 percents. Thus, it is meaningful to consider polygonal regions bounded by $k \cdot 45^{\circ}$ lines. We now assume that polygon edges are decomposed into four sets of $0^{\circ}, 45^{\circ}, 90^{\circ}$ and $135^{\circ}$ lines, respectively. Thus, the set of minimally effective chords is decomposed into four sets $\mathrm{E}_{1}, \mathrm{E}_{2}, \mathrm{E}_{3}$, and $\mathrm{E}_{4}$ of $0^{\circ}, 45^{\circ}, 90^{\circ}$, and $135^{\circ}$ lines, respectively. This implies that the intersection graph is a 4-partite graph since any two minimally effective chords $s^{\prime}$ and $s^{\prime \prime}$ in the same set $E_{i}$ are independent. The complexity of the maximum independent set problem for 4-partite graphs is not known, but a maximum independent set of a bipartite graph can be found in $O\left(\mathrm{n}^{5 / 2}\right)$ by a maximum matching algorithm [10]. This polynomialtime exact algorithm leads to the following approximation algorithm $\mathrm{F}$ on the maximum independent set (MIS) problem for 4-partite graphs.

\section{[Approximation algorithm $\mathrm{F}$ for MIS problem]}

Input. Four-partite graph $G=\left(V_{1}, V_{2}, V_{3}, V_{4}, A\right)$, where each node set $V_{i}$ $(i=1,2,3,4)$ corresponds to the set $E_{i}$.

Output. Independent set $I(F)$.

Method. For each pair $\left(V_{i}, V_{j}\right), i<j$, we construct the subgraph $G_{i j}$ induced by $V_{i} \cup V_{j}$. Since $G_{i j}$ is a bipartite graph, we can find a maximum independent set $I_{i j}^{*}$. Let $I(F)$ be a maximum one among $I_{i j}, 1 \leq i<j \leq 4$.

Lemma 15. The absolute performance ratio of the algorithm $F$ for the maximum independent set problem on 4-partite graphs is $1 / 2$.

Proof. Let $I *$ be a maximum independent set of a given 4-partite graph $G=\left(V_{1}, V_{2}, V_{3}, V_{4}, A\right)$ and $\alpha_{i}=\left|I * \cap V_{i}\right|$ for $i, i=1,2,3,4$. We can assume without loss of generality that $\alpha_{1} \geq \alpha_{2} \geq \alpha_{3} \geq \alpha_{4}$. Then, the maximum independent set $\mathrm{I}_{12}^{*}$ of the subgraph induced by $\mathrm{V}_{1} U \mathrm{~V}_{2}$ satisfies

$$
\left|I_{12}^{*}\right| \geq\left|I * \cap V_{1}\right|+\left|I * \cap V_{2}\right|=\alpha_{1}+\alpha_{2} \text {. }
$$

Thus, we have

$$
\left|I *{ }_{12}\right| /|I *| \geq\left(\left|I * \cap V_{1}\right|+\left|I * \cap V_{2}\right|\right) /|I *|=\left(\alpha_{1}+\alpha_{2}\right) /\left(\alpha_{1}+\alpha_{2}+\alpha_{3}+\alpha_{4}\right) \geq 1 / 2 .
$$


The set of minimally effective chords of such a polygonal region $\mathrm{P}^{\text {' can }}$ be obtained in $O(n \log n)$ time by the so-called plane sweep method. A maximum independent set of the intersection graph of those minimally effective chords of $P$ which have only two kinds of slopes can be obtained in $O\left(n^{3 / 2} \log n\right)$ time [12]. Thus, Approximation algorithm $F$ only requires $0\left(n^{3 / 2} \log n\right)$ time. Using this approximation algorithm $F$ and Theorem 9 , we have the following theorem.

Theorem 11. There is an $0\left(n^{3 / 2} \log n\right)$ time approximation algorithm for the minimum partition problem of polygonal regions with $\mathrm{k} \cdot 45^{\circ}$ $(\mathrm{k}=0,1,2,3)$ lines into trapezoids whose absolute performance ratio is two ( $3 / 2$ for polygonal regions without windows).

Other examples of an application of the approximation method A are as follows. Consider a polygonal region $P$ whose windows (internal polygons) are all rectilinear. Then the intersection graph $G(P)$ of minimally effective chords of $P$ is divided into two subgraphs $G_{1}$ and $G_{2}: G_{1}$ is the intersection graph of those minimally effective chords of $P$ which connect two vertices on the external polygon; and $G_{2}$ is the intersection graph of those minimally effective chords of $\mathrm{P}$ which connect a vertex on a window and a vertex on a window or the external polygon. Note that $G_{1}$ is a circle graph and $G_{2}$ is a bipartite graph. Thus, we can obtain a maximum independent set $I_{1}$ of $G_{1}$ in $O\left(n^{2}\right)$ time and a maximum independent set $I_{2}$ of $G_{2}$ in $O\left(n^{3 / 2} \log n\right)$ time [12]. Since a maximum independent set $I *(P)$ of $G(P)$ satisfies $1 / 2|I *(P)| \leq \max \left\{\left|I_{1}\right|,\left|I_{2}\right|\right\}$, we can obtain an $0\left(n^{2}\right)$ time approximation algorithm with absolute performance ratio $1 / 2$. Thus, we have an $O\left(n^{2}\right)$ time approximation algorithm with the absolute performance ratio 2 for partitioning a polygonal region with rectilinear windows into trapezoids.

Also consider a rectilinear region $\mathrm{P}$. An approximation algorithm with absolute performance ratio $1 / 2$ for the maximum independent set problem on bipartite graphs can be trivially obtained. Thus, we have an $O(n \log n)$ time approximation algorithm with the absolute performance ratio 2 for partitioning a rectilinear region into rectangles.

Finally, we give a remark on approximation algorithms. For a polygonal region $\mathrm{P}$, let $|\mathrm{I} *(\mathrm{P})|=\mathrm{a}(\mathrm{P}) \mathrm{n}(\mathrm{P})$, where $\mathrm{a}(\mathrm{P})$ is a parameter. Clearly, $0 \leq$ $\mathrm{a}(\mathrm{P}) \leq 1 / 2$. In practice, $\mathrm{P}$ contains not so many minimally effective chords, and consequently, $a(P)$ is very small. For example, current VLSI artwork data may satisfy $a(P) \leq 1 / 10$. Thus, it is meaningful to analyze the worst case ratio of an approximation algorithm with $a(P)$ as a parameter. By an 
analysis similar to the one described above, we can obtain an approximation algorithm with worst case ratio $b(P)=1+(4 a(P) / c(3-4 a(P)))$ for a polygonal region $\mathrm{P}$ if we have a (1-1/c) approximation algorithm for the maximum independent set problem on intersection graphs of minimally effective chords of polygonal regions. Note that if $a(P)=1 / 2$ then $b(P)=1+2 / c$ and if $a(P)=1 / 8$ then $b(P)=1+1 / 5 c$. Thus, Partition method $H(c=1)$ may be a good approximation algorithm in practice.

\section{Conclusion.}

We have considered the problem of partitioning a polygonal region into a minimum number of trapezoids with two horizontal sides. We first introduced an index $t(P)=n(P)+w(P)-h(P)-1$ of a polygonal region $P$, where $n(P), w(P)$, and $h(P)$ are the number of vertices, windows, and horizontal edges of $P$, respectively. Owing to this index, we could have many results rather easily. Investigating the effect of a chord by employing this index, we showed that the minimum number $M *(P)$ of trapezoids is given by $M *(P)=t(P)-d(P)=n(P)+w(P)-h(P)-d(P)-1$, where $d(P)$ is the cardinality of a maximum independent set of the intersection graph of minimally effective chords of $P$. Thus, the problem is polynomially equivalent to the problem of finding a maximum independent set of an intersection graph of minimally effective chords of P. Next, we showed that the class of intersection graphs of minimally effective chords of polygonal regions is the class of straight-lines-in-the-plane graphs. Since the problem of finding a maximum independent set of a straight-lines-in-the-plane graph has been shown to be NP-complete [13], so is this problem of partitioning a polygonal region into a minimum number of trapezoids. Although the problem of partitioning a polygonal region into a minimum number of trapezoids is NP-complete, there is a polynomial-time algorithm for partitioning a polygonal region with a fixed number of windows into a minimum number of trapezoids. In fact, we have presented an $O\left(n^{2}\right)$ time algorithm for a polygonal region $P$ without windows by employing an $\mathrm{O}\left(\mathrm{n}^{2}\right)$ time algorithm for finding a maximum independent set of a circle graph and by showing that an intersection graph of minimally effective chords of $\mathrm{P}$ is a circle graph. Also, we gave an $O\left(n^{2+w}\right)$ time algorithm for a polygonal region with w windows. Finally, we presented an $O(n \log n)$ time natural approximation algorithm which uses only horizontal chords. We showed that the absolute performance ratio of the algorithm is 3 for polygonal regions ( 2 for polygonal regions without windows). 
The approach of introducing an index of a polygonal region may be applied for the problem of partitioning a polygonal region into another fundamental figures. For example, we have obtained an $0(n \log n)$ time algorithm with the absolute performance ratio 4 for partitioning a polygonal region with windows into triangles [2].

\section{Aknowledgements}

The authors would like to thank Mr. Kunio Mori and Dr. Hiroshi Kawanishi of Nippon Electric Co. for exposing them to the problem and for their valuable comments and discussions. They also thank Mr. Masao Sato and Professor Tatsuo Ohtsuki of Waseda University for their helpful suggestions on these topics. The first and third authors would like to express their gratitude to Professor Masao Iri of the university of Tokyo for his constant guidance and encouragement.

\section{References}

[1] Asano, T. and Asano, T. Minimum partition of polygonal regions into trapezoids. Proc. 24th Annual IEEE Symp. on Foundations on Computer Science, Tucson, 1983, pp. 233-241.

[2] Asano, T., Asano, T. and Ohsuga, Y. Partitioning a polygonal region into a minimum number of triangles. Trans. IECE Japan, E67 (1984), to appear.

[3] Asano, T., Asano, T. and Pinter, R.Y. Polygon triangulation: efficiency and minimality. Submitted.

[4] Chazalle, B. and Dobkin, D. Decomposing a polygon into convex parts. Proc. 11th Annual ACM Symp. on Theory of Computing, 1979, pp.38-48.

[5] Ehrlich, G., Even, S. and Tarjan, R.E. Intersection graphs of curves in the plane. J. Combinatorial Theory Series B, 21 (1976), 8-20.

[6] Garey, M.R. and Johnson, D.S. Computers and Intractability: A Guide to the Theory of NP-completeness. W.H. Freeman and Company, San Francisco, 1979.

[7] Gavril, F. Algorithms for a maximum clique and a maximum independent set of a circle graph. Networks, 3 (1973), 261-273.

[8] Greene, D.H. The decomposition of polygons into convex parts. In: Preparata, F.P. (Ed.) Advances in Computing Research, 1

(Computational Geometry). JAI Press, London, 1983, pp. 235-259.

[9] Harary, F. Graph Theory. Addison-Wesley, Reading, Mass., 1969. 
[10] Hopcroft, J.E. and Karp, R.M. An $n^{5 / 2}$ algorithm for the maximum matchings in bipartite graphs. SIAM J. Comput., 2 (1973), 225-231.

[11] Imai, H. Finding a Maximum-Weight Independent set of an Interval Graph and a 2-circle Graph. Unpublished note, Department of Mathematical Engineering and Instrumentation Physics, University of Tokyo, 1983.

[12] Imai, H. and Asano, T. Efficient Algorithms for Geometric Graph Search Problems. RMI83-05, Department of Mathematical Engineering and Instrumentation Physics, University of Tokyo, 1983.

[13] Johnson, D.S. The column of NP-completeness: an ongoing guide. J. Algorithms, 3 (1982), 182-195.

[14] Kashiwabara, T. Algorithms for some intersection graphs. In: Saito, N. and Nishizeki, T. (eds.) Graph Theory and Algorithms, Sendai, October, 1980. Lecture Notes in Computer Science 108, Springer Verlag, Berlin, 1981, pp.171-181.

[15] Kashiwabara, T. Private communication, 1983.

[16] Keil, J.M. Decomposing Polygons into Simpler Components. Ph.D.

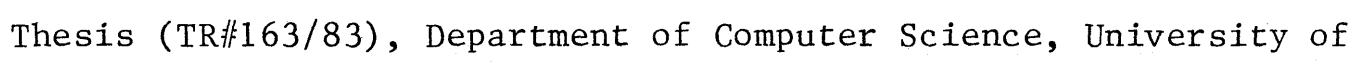
Toronto, 1983.

[17] Lichtenstein, D. Planar formulae and their uses. SIAM J. Comput., 11 (1982), 329-343.

[18] Lingas, A. The power of non-rectilinear holes. In: Nielsen, M. and Schmidt, E.M. (eds.) Ninth International Colloquium on Automata, Languages and Programming, Aarhus, July, 1982. Lecture Notes in Computer Sicence 140, Springer-Verlag, Berlin, 1982, pp.369-383.

[19] Lipski, W. Jr., Lodr, E., Luccico, F., Mugnai, C. and Pagli, L. On two dimensional data organization II. Fundmenta Informaticae, 2 (1979), 245-260.

[20] Ohtsuki, T. Minimum dissection of rectilinear regions. Proc. of 1982 IEEE International Symposium on Circuits ans Systems, Rome, 1982, pp.1210-1213.

[21] Sugiyama; N. and Saitoh, K. Electron-beam exprosure system AMDES. Computer Aided Design, 11 (1979), 59-65. 